\title{
Former les maîtres de stage à l'accompagnement en ÉPS : une approche centrée sur le codéveloppement professionnel
}

\author{
François Vandercleyen
}

François Vandercleyen.

Résumé. Afin de contribuer à la formation des maîtres de stage, une étude-pilote centrée sur l'approche de « codéveloppement professionnel (CODEV) » a été menée à l'Université de Sherbrooke. Cette approche de formation s'appuie sur une démarche structurée qui mise sur les interactions et le partage d'expériences vécues par les enseignants dans le but de les aider à améliorer leurs pratiques professionnelles. L'étude avait pour principal objectif d'identifier les bénéfices et les limites de ce modèle de formation. Un groupe de CODEV, composé de six maîtres de stage en ÉPS, s'est formé, animé par un des participants qui jouait le rôle d'animateur auprès de ses pairs. Il en résulte que «briser l'isolement», en ouvrant un espace bienveillant de dialogue et d'échanges sur les pratiques d'accompagnement, à partir du vécu réel des maîtres de stage en ÉPS, constitue un des principaux bénéfices rapportés.

Mots-clés. Maître de stage, Accompagnement, Codéveloppement professionnel

\section{Introduction}

L'UNESCO reconnaît la formation et professionnalisation des maîtres de stage 1 (MS) comme un enjeu crucial dans le domaine de l'éducation (Ria, 2015). Pour atteindre l'objectif d'une « éducation de qualité » pour tous, il est nécessaire que tous les intervenants des milieux scolaires, incluant les accompagnateurs de stage, s'engagent dans une démarche de développement continu (Desjardins, 2015).

Dans la plupart des contextes de formation, le maître de stage se voit confier un double mandat: accompagner le futur enseignant dans le développement de compétences professionnelles, ainsi qu'évaluer ces dernières. Assumer ce double mandat ne va pas de soi. Car il ne suffit pas de savoir enseigner pour savoir accompagner. Au Québec, la formation à l'accompagnement n'est pas obligatoire. Le Ministère de l'Éducation incite fortement les maîtres de stage à y participer, tandis que les universités ont le devoir de mettre à leur disposition une offre de formation. En revanche, aucune prescription ou cadre légal existe quant à la nature et aux contenus de ces formations. Si des formations à l'accompagnement de stagiaire existent, la plupart s'appuient sur des dispositifs dont les contenus sont préprogrammés et imposés aux participants (Portelance, Gervais, Lessard \& Beaulieu, 2008). Desbiens, Spallanzani et Borges (2013) soulignent à ce titre le manque de recherches sur les dispositifs de formation à l'accompagnement. Parmi ces recherches dans le domaine de l'éducation physique, Boudreau (2009) note la prédominance d'une approche «applicationniste » où le maître de stage applique des stratégies d'accompagnement proposées par le formateur, sans que ce dernier ne s'appuie nécessairement sur l'expérience et les compétences « déjà-là » des participants. Or, la grande majorité des maîtres de stage, lorsqu'ils participent à ce genre de formation, ont déjà développé une expérience d'accompagnement avec plusieurs stagiaires. Comment mobiliser et exploiter ces savoirs d'expérience?

Afin de répondre à ce questionnement, nous avons élaboré et expérimenter un dispositif de formation centré le « groupe de codéveloppement professionnel » (CODEV), développé par Payette et Champagne (1997). Cette approche peut être définie comme une pratique de formation qui mise sur les interactions entre les participants pour favoriser une amélioration de leur pratique professionnelle à partir d'une démarche structurée de réflexion sur l'action. Le CODEV trouve ses fondements au carrefour de plusieurs ancrages théoriques tels que l'action learning, le modèle praxéologique, les communautés de pratique ou encore l'apprentissage expérientiel de Kolb (1984).

L'idée maîtresse de la démarche est la suivante : mieux comprendre ensemble pour mieux agir individuellement 
Former les maîtres de stage à l'accompagnement en ÉPS : une approche centrée sur le cod...

(Champagne, 2015). Le postulat sous-jacent est que tout individu «apprend de sa propre pratique, en écoutant et en aidant des collègues à cheminer dans la compréhension et dans l'amélioration effective de leur pratique » (p. 8). Les participants réfléchissent ensemble et échangent à partir de préoccupations professionnelles vécues, où l'expérience de chacun devient une ressource mise à disposition de tous. Le moyen privilégié est une démarche structurée de consultation lors de laquelle, dans une perspective de recherche conjointe, les participants échangent afin d'enrichir leur compréhension mutuelle (Champagne \& Desjardins, 2012).

Si la dimension «sociale » est centrale, une autre dimension importante du CODEV est la structure des échanges, composée de six étapes successives, dont voici le déroulement (Payette \& Champagne, 1997) :

1. Exposé d'une situation professionnelle par un des participants (le «narrateur »);

2. Clarification de la situation par les membres du groupe («participants »);

3. Identification du besoin du « narrateur » qui fait l'objet de la consultation ;

4. Analyse de la situation par les «participants » (échanges, suggestions, pistes);

5. Synthèse et pistes d'action retenues par le «narrateur »;

6. Évaluation de la démarche et bilan des apprentissages réalisés par l'ensemble des participants.

Afin d'assurer le bon fonctionnement du groupe de CODEV, il importe de distinguer trois rôles principaux : l'animateur, le narrateur et les participants. L'animateur a pour mandat d'aider le groupe à cheminer à travers les six étapes et est le garant de la démarche de consultation. Le rôle de l'animateur est principalement d'assurer une dynamique de groupe (gestion des interactions, du tour de parole, du temps, etc.) et de mettre en place un climat de confiance. Le narrateur est le porteur de la situation professionnelle qui fera l'objet de la consultation. Lors de la première étape, il expose un épisode professionnel qui le préoccupe et dont il souhaite avoir le point de vue du groupe. Les autres participants se mettent alors au service du narrateur pour l'aider à réfléchir à sa situation problématique et l'aider à trouver des pistes de solutions. Le groupe de CODEV se distingue ainsi clairement d'un "focus group ", et doit davantage être considéré comme une communauté d'apprentissage (Payette \& Champagne, 1997).

Sous la forme d'un projet-pilote de recherche-action en collaboration avec les milieux scolaires, un dispositif de formation centré sur l'approche de codéveloppement professionnel a ainsi été implanté à l'Université de Sherbrooke, pour coformer les accompagnateurs de stagiaires en enseignement. Plusieurs groupes ont été mis en place dans différentes disciplines scolaires, dont un groupe spécifique en éducation physique et à la santé. L'objectif de l'étude est double : (1) identifier les bénéfices et les limites de ce dispositif déclarés par les maîtres de stage en ÉPS participants ; (2) décrire et comprendre les différents enjeux, les défis et les conditions inhérentes à ce dispositif.

\section{Méthodologie}

De nature qualitative, la présente recherche s'inscrit dans une visée à la fois exploratoire et compréhensive (Paquay, Crahay \& De Ketele, 2006). Le recrutement des participants, les modalités de collecte et d'analyse de données sont précisés. Deux profils de participants ont été recrutés pour participer à l'étude : un maître de stage dit «ressource » (MSR) qui a assumé le rôle d'animateur du groupe de codéveloppement professionnel en ÉPS ; et des maîtres de stage "participants ». Le premier est un enseignant d’EPS expérimenté au secondaire, maître de stage depuis une dizaine d'années et superviseur universitaire, pour le programme du BEÉPS à l'Université de Sherbrooke. Celui-ci a suivi une formation à l'animation de groupes de CODEV dispensée par le collectif de recherche afin de s'approprier son rôle. C'est aussi lui qui s'est chargé de l'organisation et du recrutement des participants. Les seconds, les maîtres 
Actes de la 11ème Biennale de l'ARIS : Former des citoyens physiquement éduqués. Axe 2 - Vers une éducation physique de qualité, 10.25518/sepaps20.360

de stage participants (MSP) sont six enseignants en ÉPS, du primaire $(n=2)$ et du secondaire $(n=4)$, de la région de l'Estrie (Québec). Recrutés sur une base volontaire, ces mêmes MS ont accepté de participer librement à une rencontre d'une durée de $2 \mathrm{~h} 30$. Conformément à la démarche de CODEV, un des MS participants (le narrateur) s'est prêté au jeu d'exposer une situation professionnelle interpellante aux autres. La situation portait sur un manque de professionnalisme d'un stagiaire (éthique de travail). Dans ce cas, il s'agissait d'un épisode d'accompagnement vécu par le passé et non résolu. Trois types de données ont été récoltées : (1) une entrevue avec les participants avant et après les rencontres de groupe ; (2) des audio-enregistrements des interactions entre les participants ; (3) des bilans individuels d'apprentissage et d'appréciation de la démarche réalisés à la fin des rencontres. Après transcription sous la forme de verbatim, l'ensemble des données compilées a fait l'objet d'une analyse de contenu selon un modèle ouvert (L'Écuyer, 1987).

\section{Résultats et discussion}

À des fins de concision, les résultats sont présentés et discutés comme suit : (1) les bénéfices et les apprentissages perçus les maîtres de stage en ÉPS participants ; (2) les limites qu'ils perçoivent; (3) les enjeux, défis et conditions de mises en œuvre de groupe de codéveloppement professionnel à l'accompagnement de stagiaires en ÉPS observés.

\section{Bénéfices et apprentissages perçus par les maîtres de stage en ÉPS}

Souvent esseulés dans leurs pratiques d'accompagnement, les MS en ÉPS estiment de façon unanime que de participer à un groupe de codéveloppement professionnel a permis de «briser l'isolement» en ouvrant un espace de dialogue bienveillant avec leurs pairs. En effet, il n'existe pas ou peu de lieu formel d'échanges autour de cette fonction et, quand ils ont lieu, ceux-ci sont souvent anecdotiques. «Prendre le temps de se poser » pour partager leur expérience, discuter et confronter leurs points de vue s'est avérer également bénéfique selon les participants.

Un autre bénéfice que tous reconnaissent consiste à témoigner de leur vécu professionnel, par définition singulier, plutôt que de l'accompagnement «en général ». Une des forces du CODEV est effectivement de partir d'une situation problématique ou préoccupante réellement vécue par un des participants. Cette situation initiale, chargée émotivement, agit comme un déclencheur ou un catalyseur de questionnement professionnel auprès des autres. En écho, les maîtres de stage ont tendance à s'identifier et à se reconnaître collectivement dans cette situation (Vandercleyen et al., 2019). Plusieurs MS participants admettent d'ailleurs qu'une véritable « communauté » est née à la suite de cette expérimentation. La plupart des maîtres de stage participants ont également exprimé l'importance de se retrouver entre enseignants de la même discipline : «Entre nous, on se comprend, C'est plus facile qu'avec les profs titulaires (...) notre réalité est différente ». Se rassembler entre MS, exclusivement en ÉPS, répondrait à un besoin d'appartenance sociale (Richer \& Vallerand, 1998).

Au-delà de la dimension collective, la démarche de CODEV a permis aux maîtres de stage de faciliter la mise en mots de leurs pratiques individuelles, en dévoilant leurs savoirs d'expérience (Dumoulin \& Desjardins, 2019). Selon certains MS, cette verbalisation provoquée a permis de nouvelles prises de conscience voire des remises de question, notamment de leurs conceptions en lien avec l'accompagnement ( Je vois mon rôle différemment maintenant »; «Je repars avec davantage de questions que de réponses ») mais aussi le développement de nouvelles stratégies ( J'ai appris comment gérer un conflit avec mon ou ma stagiaire si j'avais à vivre une situation similaire »). Un des participants a quant à lui tenu à souligner le "climat de confiance où la solidarité est très présente ». Après la rencontre, un des MS qui avait joué le rôle du «narrateur » a eu ces mots auprès des autres membres : «Merci du fond du cœur, vous m'avez si généreusement aidé dans mon besoin de guérir et résoudre une page sombre de mon passé en tant qu'enseignant associé !». Sans avoir de visée thérapeutique, ce message en dit long néanmoins sur l'effet cathartique du CODEV. Plus largement, le groupe de CODEV a permis aux maîtres de stage de mesurer toute la complexité de l'acte d'accompagner 
Former les maîtres de stage à l'accompagnement en ÉPS : une approche centrée sur le cod...

(Dumoulin \& Desjardins, 2019 ; Vandercleyen, L’Hostie \& Dumoulin, 2019).

\section{Limites perçues par les maîtres de stage en ÉPS}

Si les maîtres de stage en ÉPS participants reconnaissent l'importance de "s'arrêter pour échanger », ils dénoncent par ailleurs la difficulté de «se libérer » de leur temps de travail pour s'engager dans ce type de démarche. La durée optimale est aussi questionnée : «Deux heures, c'est court et en même temps, c'est difficile à rentrer dans notre horaire ». Si certains ont mentionné le caractère «dynamique et convivial» de la formule, d'autres estiment que le groupe de CODEV constitue un cadre trop "structurant» voire "rigide», en laissant peu de places aux "échanges libres et spontanés ». D’autres encore plaident pour une démarche accélérée, qui permettrait à chaque participant de passer par le rôle de «narrateur ». Enfin, une minorité dit vouloir repartir avec des stratégies concrètes, plutôt que de «pelleter des nuages».

\section{Enjeux, défis et conditions de mise en œuvre des groupes de CODEV}

Le principal enjeu constaté concerne la capacité de recrutement et de rétention des maîtres de stage, pour assurer la pérennité du dispositif. Si plusieurs MS sont intéressés à s'engager dans une telle démarche, l'accompagnement des stagiaires n'est pas nécessairement leur priorité. De façon légitime, l'apprentissage et le bien-être des élèves demeure leur objectif premier. Participer à ce type de démarche n'est donc pas la formation continue qu'ils ont tendance à priorise. Parmi les défis observés, un des plus importants concerne l'appropriation de la démarche de CODEV et plus particulièrement le changement de posture qu'elle suppose. La position d'expert dans laquelle se placent certains MS, à la recherche d'une reconnaissance légitime, ne leur permettent pas de se mettre dans une position d'ouverture voire de vulnérabilité : «Ça va, je me rends compte que je ne suis pas dans le champ avec ma façon de faire... ». Le doute permet le déploiement d'une démarche d'enquête, indispensable dans l'optique d'un développement professionnel (Dumoulin \& Desjardins, 2019). Une des conditions de mise en œuvre des groupes de CODEV consiste, pour l'animateur, à installer un climat de confiance suffisant pour permettre aux participants de se mettre à nu, sans tabou.

\section{Conclusion}

Le groupe de codéveloppement professionnel apparaît comme une approche de formation fertile qui permet l'essor d'une réflexion à la fois individuelle et collective par les maîtres de stage en ÉPS sur leurs propres pratiques d'accompagnement de stagiaires. Le principal bénéfice qu'ils perçoivent est un espace d'échanges structurés à partir de leur expérience permettant d'expliciter et de légitimer leurs pratiques d'accompagnement.

1 Le maître de stage fait référence ici à l'enseignant qui accepte d'accueillir et d'accompagner un stagiaire en formation à l'enseignement dans sa...

\section{Bibliographie}

Boudreau, P. (2009). Pour un modèle de supervision de type inductif en formation à la supervision de maîtres de stage en éducation physique. Éducation et Francophonie, 37(1), 122-141.

Champagne, C. (2015). Le groupe de codéveloppement professionnel, pour être partenaires dans l'apprentissage et le développement professionnel. Le Partenaire, 24(2), 8-12.

Champagne, C., \& Desjardins, M. (2012). Le groupe de codéveloppement professionnel : une approche puissante et de plus en plus reconnue. Effectif, 17(1), 14-21. 
Actes de la 11ème Biennale de l'ARIS : Former des citoyens physiquement éduqués. Axe 2 - Vers une éducation physique de qualité, 10.25518/sepaps20.360

Desbiens, J-F., Spallanzani, C., \& Borges, C. (2013). Quand le stage en enseignement déraille : regards pluriels sur une réalité trop souvent occultée. Québec : PUQ

Desjardins, J. (2015). Stimuler l'épanouissement d'une culture de la formation continue au Québec : visée réaliste ou fantasmatique? In O. Maulini, J. Desjardins, R. Etienne, L. Paquay (Éds.), À qui profite la formation continue des enseignants? (pp. 69-92). Bruxelles : De Boeck.

Dumoulin, M-J., \& Desjardins, J. (2019). Un modèle de codéveloppement professionnel en formation continue des enseignants associés au Québec : comment déjouer l'opposition entre reconnaissance et mise en question du savoir d'expérience? In P. Guibert, X. Dejemeppe, J. Desjardins, O. Maulini (Éds.). Questionner et valoriser le métier d'enseignant. (pp. 157-178). Bruxelles : De Boeck.

Kolb, D.A. (1984). Experiential learning. Englewood Cliffs (N-J): Prentice-Hall.

L'Écuyer, R. (1987). L’analyse de contenu : notions et étapes. In J-P. Deslauriers (Éd.). Les méthodes de la recherche qualitative. (pp. 49-65). Québec : PUQ.

Paquay, L., Crahay, M., \& De Ketele, J-M. (2006). L’analyse qualitative en éducation. Bruxelles : De Boeck.

Paul, M. (2004). L'accompagnement : une posture professionnelle spécifique. Paris : L'Harmattan.

Payette, A., \& Champagne, C. (1997). Le groupe de codéveloppement professionnel. Québec : PUQ.

Portelance, L., Gervais, C., Lessard, M. \& Beaulieu, P. (2008). Cadre de référence pour la formation des enseignants associés et des superviseurs. Rapport de recherche, MELS, Québec.

Ria, L. (2015). Former les enseignants au XXI ${ }^{e}$ siècle. Bruxelles : De Boeck.

Richer, S. F., \& Vallerand, R. J. (1998). Sentiment d'appartenance sociale. Revue Européenne de Psychologie Appliquée, 48, 129-137.

Vandercleyen, F., L'Hostie, M., \& Dumoulin, M-J. (2019). Le groupe de codéveloppement professionnel pour former à l'accompagnement de stagiaires : conditions, enjeux et perspectives. Québec : PUQ.

\section{Notes}

1 Le maître de stage fait référence ici à l'enseignant qui accepte d'accueillir et d'accompagner un stagiaire en formation à l’enseignement dans sa classe (ou son gymnase) pour une période déterminée.

PDF automatiquement généré le 2023-04-26 14:42:12

Url de l'article : https://popups.uliege.be/sepaps20/index.php?id=360

Publié par ULiège Library en Open Access et distribué suivant les termes et les conditions de la licence CC-BY

(https://creativecommons.org/licenses/by/4.0/deed.fr) 